\title{
In Vivo Confocal Raman Microspectroscopy of the Skin: Effect of Skin Care Products on Molecular Concentration Depth-Profiles
}

\author{
Shuliang L. Zhang*, Peter J. Caspers** and Gerwin J. Puppels** \\ * Unilever Research and Development, 40 Merritt Blvd., Trumbull, CT 06611 \\ **Erasmus-university Medical Center and River Diagnostics B.V., Rotterdam, The Netherlands
}

In vivo confocal Raman spectroscopy has emerged in recent years as a useful tool to obtain detailed information about the molecular composition of the skin [1-3]. It can provide information on important molecules such as water and natural moisturizing factors as well as exogenous molecules such as glycerol delivered from skin care products.

We conducted a pilot study with two subjects to evaluate the capability of the in vivo Raman technique. The study was approved by the Institutional Review Board of Unilever and an informed consent was obtained from both subjects prior to the study. The study was conducted on River Diagnostics' Skin Composition Analyzer (Model 3510 SCA). A moisturizing liquid cleanser and leave-on test product both containing glycerol were used. Three test sites, approximately $4 \times 4 \mathrm{~cm}^{2}$ each, were marked with a skin marker pen on the volar forearm. The cleanser product was applied twice daily for 7 consecutive days, while the leave-on product was applied only once, with the detailed protocol similar to that of other studies [4].

Figure 1 shows a set of Raman spectra of the skin and the spectral regions used to obtain the water content in the skin normalized to that of the proteins. Detailed calculation and calibration for water content can be found elsewhere [1]. Figure 2 shows the water concentration profiles on each test site at baseline. There is a clear difference between the test sites within each arm and between the two subjects. All three sites started with the similar water content on the top of the stratum corneum (SC) at $20 \%$ and $25 \%$ for subjects 1 and 2, respectively. The water content rose through the SC and all leveled off at nearly $70 \%$ in the viable epidermis but at different depths between the test sites within a subject and between the two subjects.

Figure 3 shows the result from the applications of the cleansing product. The noisier spectrum is the difference spectrum of the post product application from the baseline. The average spectra over the first $5 \mu \mathrm{m}$ of the skin were used to obtain the difference spectrum. The two component spectra are the fitted contributions from the cleanser and water. This indicates that the treated skin has a surplus of water over the baseline skin, which is consistent with the increased water content from the water profiles (not shown).

For the glycerol penetration study, deuterated glycerol (glycerol-d5) was used in order to enhance the detection sensitivity and to differentiate the exogenous glycerol from the endogenous compound. Figure 4 shows a series of Raman spectra at different depths of the skin at one location measured 3 hours after the product application. Glycerol signals are clearly visible from these spectra. Similar spectra were observed at other locations within the test site. The relative peak intensities of glycerol can be plotted as the depth of the penetration to generate the depth profiles as shown in Figure 5.

In summary, in vivo confocal Raman microspectroscopy showed a great promise in this pilot study for measuring delivery and penetration of important skin cosmetic ingredients and their effect on skin properties as measured in the changes in skin composition. Large-scale clinical studies are needed to validate the findings. 


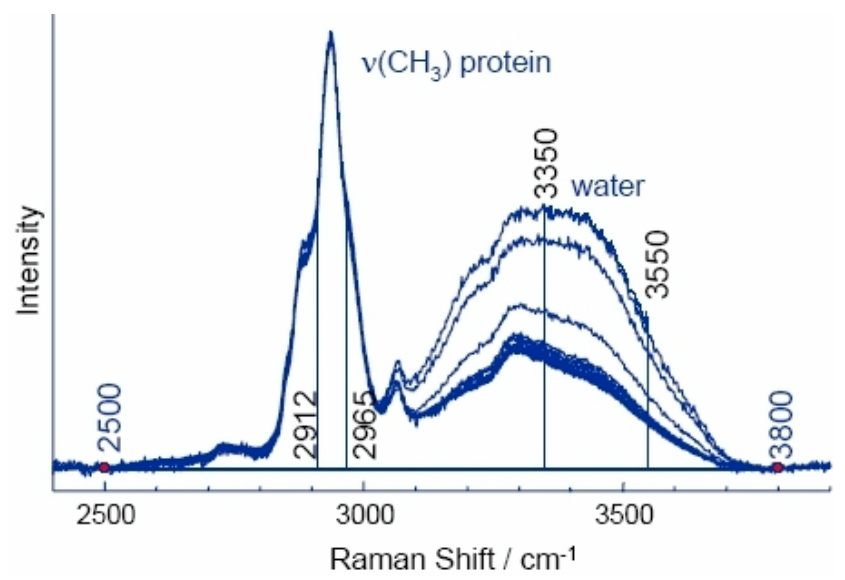

\section{References}

[1] P. J. Caspers, G. W. Lucassen , H. A. Bruining, and G. J. Puppels, J. Raman Spectrosc. 31 (2000) 813.

[2] P. J. Caspers, G. W. Lucassen, E. A. Carter, H. A. Bruining, and G. J. Puppels, J. Invest. Derm. 116 (2001) 434.

[3] P. J. Caspers, G. W. Lucassen, and G. J. Puppels, Biophys. J. 85 (2003) 572.

[4] S. L. Zhang, C. L. Meyers, K. Subramanyan and T. M. Hancewicz, J. Biomed. Optics, in press (2005).

Figure 1. Raman spectra of the skin in the region of the $\mathrm{C}-\mathrm{H}$ and $\mathrm{O}-\mathrm{H}$ stretching vibrations and determination of water content based on the ratio of the integrated $\mathrm{O}-\mathrm{H}$ intensity of water between 3350 and $3550 \mathrm{~cm}^{-1}$ to that of the C-H intensity of proteins between 2912 and $2965 \mathrm{~cm}^{-1}$. The 2500 and $3800 \mathrm{~cm}^{-1}$ points are used to define the spectral baseline.
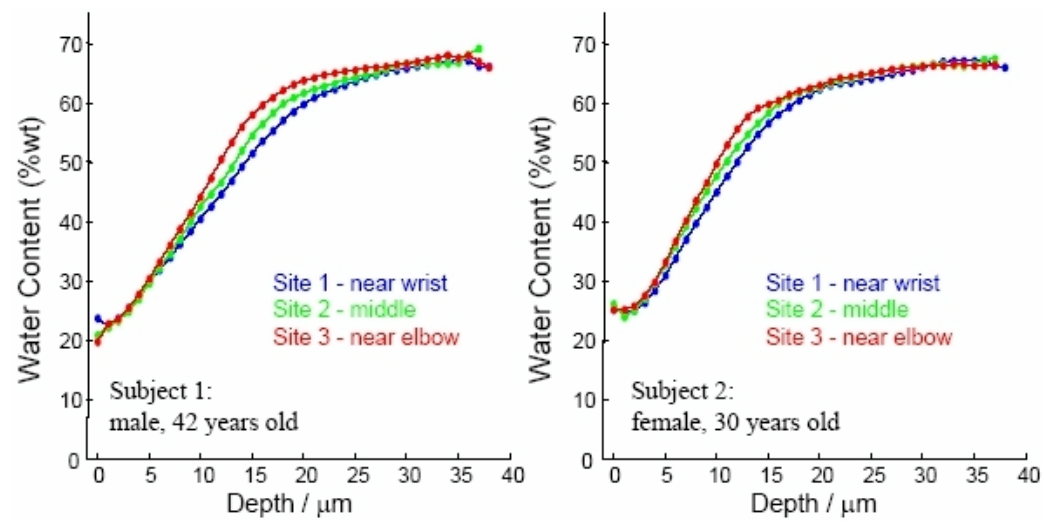

Figure 2. Water concentration profiles at baseline.

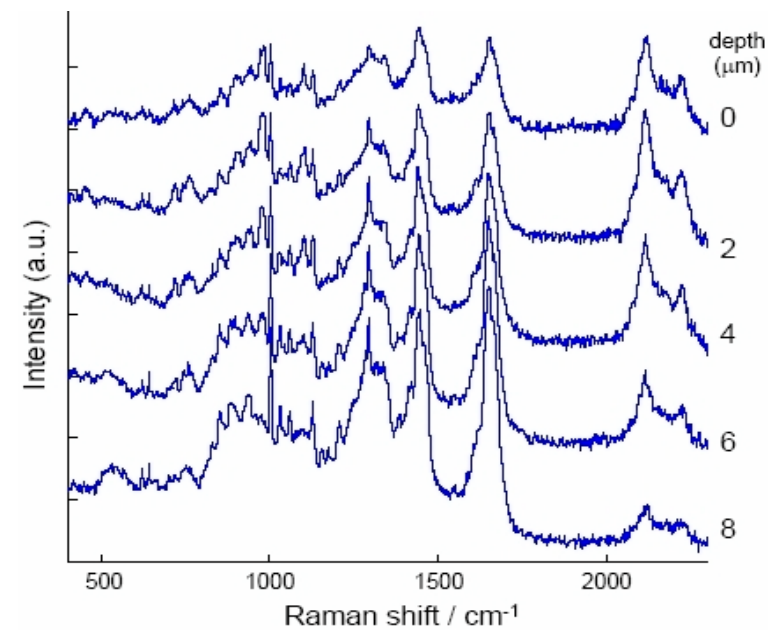

Figure 4. Raman spectra measured at different depths at one measurement location at 3 hours after an application of the deuterated glycerol-containing test product. The surface was cleaned with wet cotton prior to the measurement. The spectra are offset from each other for clarity. The bands above $2000 \mathrm{~cm}^{-1}$ are due to the C-D stretching vibrations from the deuterated glycerol.

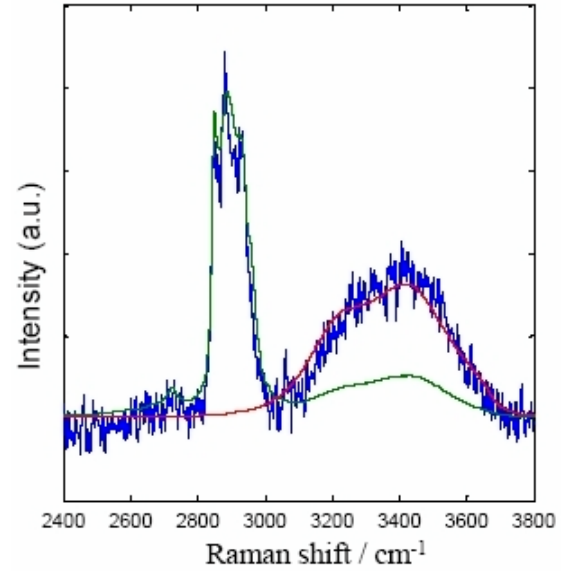

Figure 3. The result from the cleanser. The difference spectrum was grossly modeled with the spectra of the cleanser (green) and water (red).

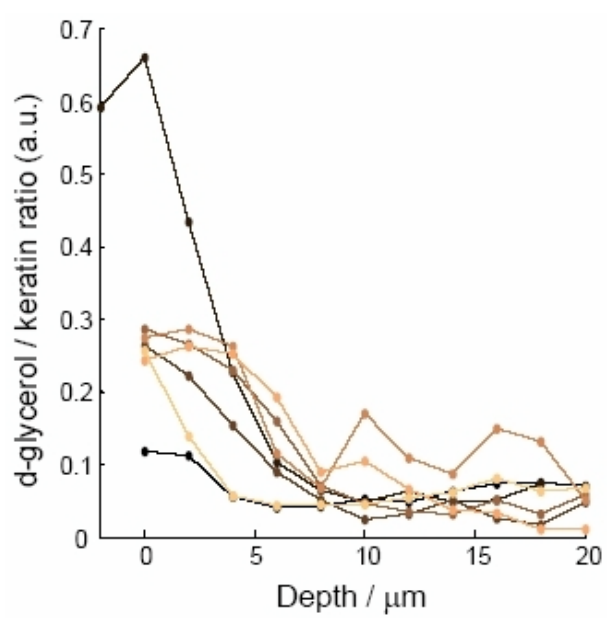

Figure 5. The glycerol penetration depth profiles at 3 hours after the product application. 